\title{
Measurement Issues in Tests of the Socioecological Complexity Hypothesis
}

\author{
Jordan Lasker \\ Texas Tech University \\ John D. Haltigan \\ University of Toronto \\ George B. Richardson \\ University of Cincinnati
}

Please address correspondence to Dr. George Richardson, School of Human Services, College of Education, Criminal Justice, and Human Services [460R Teachers-Dyer Complex; george.richardson@uc.edu; 513-556-9261].

* This paper was accepted for publication in Evolutionary Psychological Science on 09/17/2021. This is the pre-copy edited, peer-reviewed version. * 
Abstract

Recent research has advanced a socioecological theory to account for differences in the strengths of covariances among disparate personality measurements in different cultures. According to this socioecological complexity hypothesis, niche diversity is greater in more complex societies and this relaxes the covariances among personality traits (e.g., see Lukaszewski et al., 2017). While the socioecological complexity hypothesis is novel and interesting, we suggest that approaches used to test it thus far are conceptually and methodologically flawed. Accordingly, extant findings should be considered cautiously and not construed as evidence against alternative explanations for differences in personality or other behavioral trait covariances within or across countries. To advance the literature, here we review measurement issues that require attention in efforts to test the socioecological complexity hypothesis and then describe approaches that may aide researchers in overcoming them.

Keywords: Big Five, measurement invariance, personality, behavioral ecology, environment 


\section{Measurement Issues in Tests of the Socioecological Complexity Hypothesis}

Drawing on a functionalist and cultural anthropological framework, Lukaszewski,

Gurven, von Rueden and Schmitt (2017) introduced a novel socioecological complexity hypothesis to explain the emergence of different patterns of personality and behavioral trait covariance across disparate cultures. In short, the socioecological hypothesis (Lukaszewski et al., 2017; see also Smaldino, Lukaszewski, von Rueden \& Gurven, 2019) is based on the idea that personality and behavioral traits will covary if they are influenced by the same functionalist motivations (e.g., status pursuit, self-protection) and that different patterns of trait covariation will emerge as a function of socioecological contingencies in the attainment of functional objectives. At the heart of these considerations is the idea that the degree of personality trait covariation observed within a society will be inversely related to its socioecological complexity, with this concept representing the degree of "niche specialization within large-scale cooperative groups and institutions” (Lukaszewski et al., 2017, p. 2). In Lukaszewski et al.’s (2017) publication, socioecological complexity was represented by three measures that should theoretically be related to it including the Human Development Index, urbanization levels, and sectoral diversity, which is the variety of products able to be produced by a country (see Lukaszewski et al., 2017 for further details). To assess how socioecological complexity affected the covariances of personality traits, Lukaszewski et al. (2017) drew on data from the Big Five Inventory (BFI; Benet-Martinez \& John, 1998), a self-report personality measurement instrument intended to tap the constructs agreeableness, conscientiousness, emotional stability, extroversion, and openness to experience, across 55 countries. They observed that the scores for those personality traits covaried more tightly in less socioecologically complex countries and interpreted this result as support for their socioecological complexity hypothesis. 
In our view, a key problem with the approach used by Lukaszewski et al. (2017), as well as more recent work based on their hypothesis (Durkee et al., 2020; Smaldino et al., 2019), is the use of unit-weighted scores despite clear evidence that these scores did not have the same meanings across countries (i.e., 'measurement invariance' or MI; Meredith, 1993). Superficial attention to measurement concerns in these studies, and more specifically, violations of MI, appears to have precluded researchers from producing evidence with which the socioecological complexity theory could be evaluated. In what follows, we discuss the assumptions of unitweighted summary scores, the stages of MI testing as they are commonly understood in the literature (e.g., Meredith, 1993; Meredith, 1995; DeShon, 2004), and we identify ways in which measurement bias may have compromised comparisons of covariances among the Big Five factors. We advise that future attempts to evaluate the socioecological complexity hypothesis should be undertaken from a clearer position regarding the theoretical status of latent variables (e.g., Borsboom, Mellenbergh, \& van Heerden, 2003) and devote greater attention to conceptualanalytical coherence (e.g., Collins, 2006). Following these discussions, we attempt to advance the literature by describing approaches that may aide researchers in testing evolutionary theories concerning personality trait covariation differences across groups.

\section{Implications of MI for Evaluation of the Socioecological Hypothesis: A Critique}

MI is important for evaluating the socioecological complexity hypothesis because unless the target personality measures used to evaluate it demonstrate MI across nations, the differential patterns of personality covariation across nations may be due to measurement bias instead of differences in covariance structure among personality traits. It is important to recognize that 'personality' measurement corresponds to the measurement of factors modeled in a factor analysis, not individual observed variables (i.e., the items). If observed variables are construed as measuring personality on their own, what constitutes personality is entirely subjective. The 
reason for this is that out of the set of all potential questions to be asked regarding personality, there will inevitably be disagreement over what constitutes an item that measures a given construct and this disagreement will be insoluble on the basis of observed scores alone. This is a crucial point because it renders one unable to formally adjudicate a hypothesis such as Lukaszewski et al.'s (2017) as any interpretation of differences in the correlations of Big Five dimensions between countries as a function of socioecological complexity is likewise dubious. In contrast, by employing latent variable models, the ontological reality of latent variables can be assessed in numerous ways; for instance, by finding that all the influences on coherent sets of observed indicators are mediated by a latent variable (Edwards \& Bagozzi, 2000; Franić et al., 2013).

If latent variable models are avoided in testing the socioecological complexity hypothesis, the results should be regarded as unconfirmed because unlike in latent variable models, sum scores do not represent the constructs of theoretical interest; instead, they represent the combined influences of error, theoretically interesting constructs, bias, and so on, in potentially wildly varying degrees. This is not to say that latent variable models are by any means infallible or the only way to address this question. Indeed, they are but one way. However, the use of sum scores without assessing whether there is bias, latent variable models that are not found to fit equivalently across countries, and factor alignment without equivalent baseline (i.e., configural) model fit ${ }^{1}$ are not viable ways. The unviability of these other methods is apparent because, if groups interpret questionnaires differently, hypotheses about the structure of underlying constructs may seem confirmed, when in fact the apparently supportive results occur for reasons completely unrelated to the constructs themselves or any influence a potential

\footnotetext{
${ }^{1}$ Though this manuscript only briefly discusses issues with factor alignment, we wish to note that it can be used to force models to appear to fit in different groups if used improperly. The result of forcing a particular factor solution can be to create a factor solution that reiterates rather than removes bias.
} 
moderator may have on them. This is why interpreting a greater degree of covariance between items or sum scores intended to measure personality as evidence for a substantive hypothesis about personality cannot be considered valid when a lack of bias has not been demonstrated. We cover this in more detail below.

Assumptions of Sum Scores, The Common Factor Model, and Measurement Invariance

The use of summary scores (i.e., sums or averages) created from observed data is extremely common in the social and behavioral sciences, yet the assumptions that must be satisfied to ensure these scores are sufficient statistics (i.e., adequate summaries of item information) are rarely acknowledged. As summarized in Andersen (1977) and more recently in van der Sluis, Verhage, Posthuma \& Dolan (2010), these assumptions are:

1. unidimensionality, or local independence, the condition whereby item responses are independent net of a single latent variable,

2. expected values of the item responses have the same functional relations with the latent variable,

3. residual variance is equal for all manifest variables.

These are strong assumptions and they should be routinely checked much like the linearity and homoscedasticity assumptions in multiple regression. This is accomplished with latent variable (or common factor) models that relate a set of manifest (observed) variables to one or more latent (unobserved) variables. In these models, loadings capture the relations between manifest and latent variables, quantifying the variance in the former explicable by the latter. Latent variable models allow researchers to observe differences among loadings and residuals and gain an idea about their practical significance and the validity and fidelity of theories about the structure of a measurement instrument. If such observations do not reveal 
threats to the validity of inferences about underlying constructs of interest, it may be appropriate to use summary scores as proxies for their intended constructs.

In addition to assuming their scores were sufficient statistics, the comparison made by Lukaszewski et al. (2017) required that their scores had the same meanings across countries; in other words, they needed measurement invariance (MI). Within the common factor modeling tradition (Thurstone, 1931; 1947; Gorsuch, 1988), MI is typically assessed with factor-analytic models and can be thought of as the degree to which a measure yields the same empirical meaning and construct identity across diverse populations (e.g., racial/ethnic groups). Demonstration of MI for a given construct (e.g., extraversion) is a necessary prerequisite for evaluating group differences in means, variances, and (where relevant) residual covariances (Brown, 2006, 2015; Raykov, 2004). This is because for 'true' differences in terms of a construct to be interpreted meaningfully across groups, one must first establish that the construct in question is measured the same way in different groups (Brown, 2015).

The seminal work of Meredith (1993; see also Meredith \& Teresi, 2006) is almost universally considered the gold-standard on the topic, containing within it a detailed series of steps for testing MI. Briefly, increasingly restrictive equality constraints are imposed on the parameters of a latent variable model across different groups and measurement (in)variance is evidenced by (the lack of) deterioration in model fit (see, e.g., Liang \& Luo, 2020, for details on how model fit measures are used for model selection in MI testing). These constraints are added to the model in the following order:

(1) Configural invariance - also known as form or pattern invariance - where groups have the same model parameters; stated differently, the number of factor(s) and pattern of freed and constrained loadings and covariances is the same in each group; 
(2) Metric invariance - also known as weak invariance - assesses whether the loadings on modeled common factors are of the same magnitudes in different groups; if this holds, groups attribute the same meanings to common factors;

(3) Scalar invariance - also known as strong invariance — assesses whether groups have the same intercepts; with this degree of invariance achieved, the levels of the manifest variables have the same meanings. At this point, group means are typically considered comparable;

(4) Strict factorial invariance (SFI) - also known as omnibus or full uniqueness invariance - assesses whether groups have the same residual variances for each of their manifest variables. If this holds, the same variables are involved in producing the factor structure present in each group and measurement reliability is equal, barring differences in residual covariances.

As noted in the above description of each level, the levels of MI testing are amenable to colloquial restatement (van de Schoot, Lugtig \& Hox, 2012). Depending on the specific nomenclature referenced, there are additional stages included under the MI rubric, frequently deemed unnecessary or needlessly restrictive; these include testing whether latent variances, covariances, and factor means can also be constrained (i.e., 'structural' latent mean or variance homogeneity; cf. the alternate 'population heterogeneity' [Brown, 2006, 2015]).

With all of this said, it should be apparent that MI is crucial for cross-cultural psychological work, as, if measures do not have equivalent interpretations across groups, what is being compared could be completely irrelevant to the constructs under consideration. In what we hope is a drastic analogy, two groups being compared in terms of height might render measures in meters in one group and in kilograms in another; we can only hope some psychologist would take pause at reading mention of a group with an average height of fifty meters. Of course, it is 
also possible for violations of MI to be trivial, but this cannot be taken for granted and tests must be conducted to ascertain the degree to which bias occurs at different stages of testing. As we discuss in the section below, the case that prompted this paper likely came with a staggering degree of measurement bias.

Did Measurement Invariance Hold in Lukaszewski et al. (2017)?

Summary scores computed based on observed data require assumptions even when groups are not compared. Equal loadings and residuals for the set of items reflecting each factor are strict assumptions and violations may not always be practically significant; the effects of these violations, if found, can be assessed. Unidimensionality, however, is much more crucial. Although correctly noting the importance of MI as it pertains to evaluation of their hypothesis, Lukaszewski et al. (2017) found poor absolute fits for each configural model they fitted on the Big Five personality trait measures used in their investigation (average $\mathrm{CFI}=.15$; average RMSEA $=.230$ [90\% CI .209 - .214]); the fits they achieved were well below standard cut-offs for acceptable absolute model fit (e.g., Hu \& Bentler, 1999). At this point, pursuing further tests of the socioecological complexity hypothesis with their data using scale scores is misguided given clear evidence that one or both the unidimensionality and the loading magnitudes are noninvariant across the nations (groups) examined in their study.

Lack of unidimensionality is of key relevance to Lukaszewski et al.'s (2017) test of the socioecological complexity hypothesis, which concerns differences between groups, because it implies that MI does not hold. Below we further describe the implications of this evidence against MI for properly adjudicating whether between-nation differences in the interrelatedness of observed Big Five personality traits are entirely mediated by differences in the factor covariances. We also identify several additional issues relevant to MI that limit meaningful interpretation of existing tests of the socioecological hypothesis. 
Consider Mellenbergh's (1989) definition for MI, $f(Y \mid \eta)=f(Y \mid \eta, v)$ where $f$ is a link

function, $Y$ is a score on a manifest variable, $\eta$ is a corresponding factor score, and $v$ is the variable representing an individual's group; restated, conditional on one's position in terms of a factor score, $\eta$, their observed score, $Y$, is independent of group membership ${ }^{2}$. Importantly, this definition extends not just to groups, but to all variables; all variables must be related to an item only via the factor subsuming it. This includes the causes of a particular Big Five personality trait, future outcomes influenced by that trait, and key to Lukaszewski et al. (2017), the items used to measure other Big Five traits. This last point is important because it implies that the choice to model each personality factor independently as did Lukaszewski et al. (2017) was not a viable one. This is the case because again, for MI to be tenable, they must be able to ensure that the relationships between the different manifest variables in the correlated factor model are plausibly explained entirely by the relationships among their factors. If this is found to not be the case, MI assessment is complicated. Because no one may provide an a priori reason to believe this sort of mediation is achieved with any given dataset, they cannot justify testing isolated single-factor models. Because the modeling approach in Lukaszewski et al.'s (2017) did in fact rely on these isolated tests of single factor models, it did not allow the researchers to distinguish real differences between countries in factor covariances from differences in manifest variable residual covariances. It is possible that instead of reflecting violations of unidimensionality, the poor fits of the models in Lukaszewski et al.'s (2017) study were due to omitted cross-loadings.

\footnotetext{
${ }^{2}$ As Hood (2008) argued, this formulation of MI was problematic for the bifactor model. Restating his argument, in the bifactor case where there is a broad general factor and orthogonal factors, $\zeta$, specific to certain sets of manifest variables the equation for MI becomes $\mathrm{f}(\mathrm{Y} \mid \eta, \zeta)=\mathrm{f}(\mathrm{Y} \mid \eta, \zeta$, v), and while this may be true, it also logically implies that MI with respect to any specific factor cannot be tested because $\mathrm{f}(\mathrm{Y} \mid \eta) \neq \mathrm{f}(\mathrm{Y} \mid \eta, \zeta)$ and $\mathrm{f}(\mathrm{Y} \mid \zeta) \neq \mathrm{f}(\mathrm{Y} \mid \zeta, \eta)$ since the level of some manifest variable is the result of the influences of both $\eta$ and $\zeta$ and not either alone. Hood acknowledged that using either latent variable for grouping was strange, but the point remained: it would not be possible to test invariance with respect to either factor on its own. More importantly, to the extent that the original grouping variable, $v$, correlated with one's score on one of the latent variables, we get either $f(Y \mid \eta) \neq f(Y \mid \eta, v)$ or $\mathrm{f}(\mathrm{Y} \mid \zeta) \neq \mathrm{f}(\mathrm{Y} \mid \zeta, \mathrm{v})$ because $\mathrm{Y}$ is also affected by the unmodeled factor correlated with $\mathrm{v}$.
} 
Below we describe in detail the problem cross-loadings can pose for tests of the socioecological complexity hypothesis.

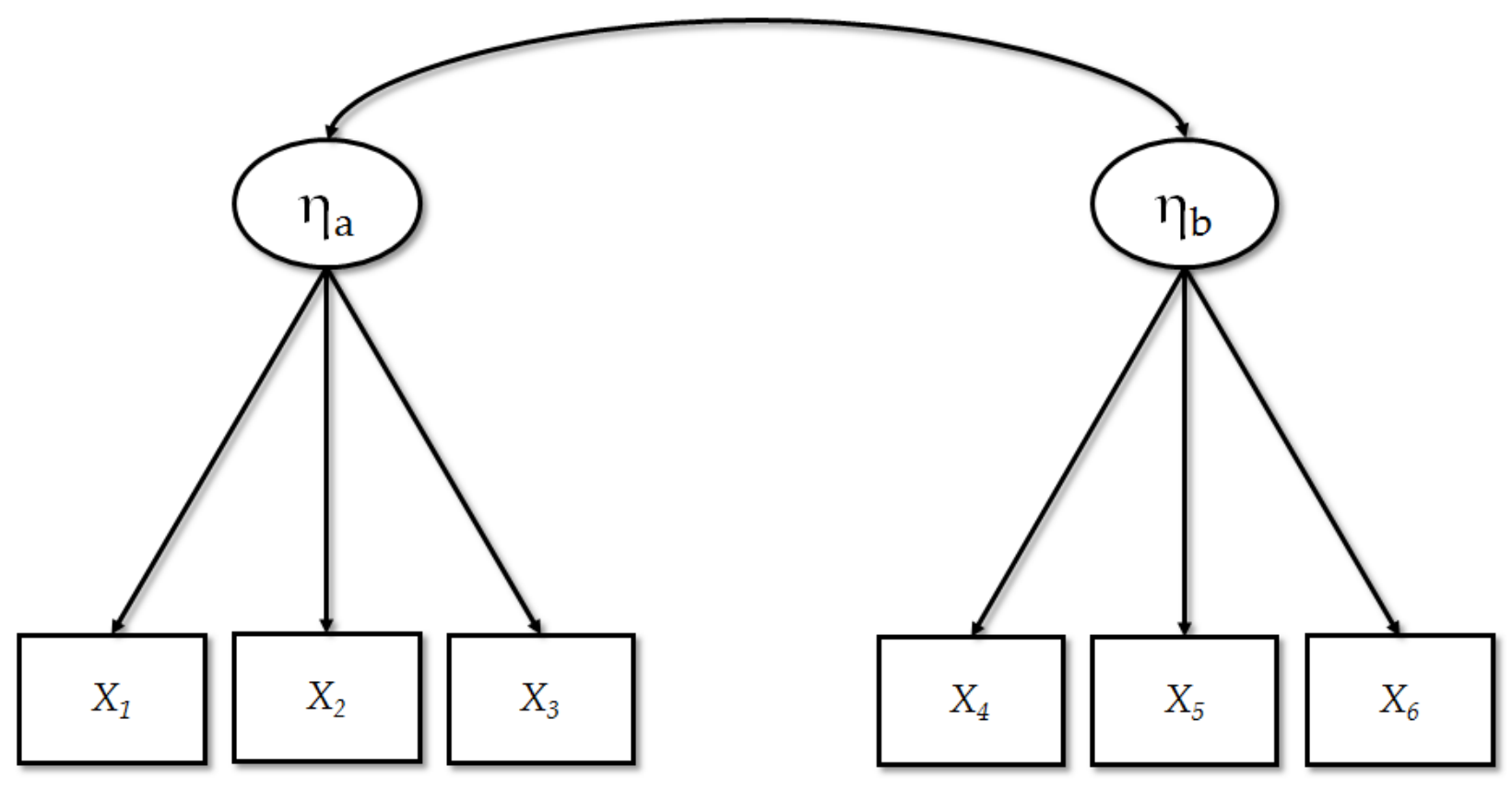

Figure I

Figure I illustrates a simple two-factor model for an example where statistical power is assumed to be sufficient and local independence is assumed to result from the positively correlated common factors $\eta_{a}$ and $\eta_{b}$. Due to this positive factor covariance, it can be inferred that all of the variables, $\mathrm{X}_{1}$ through $\mathrm{X}_{6}$, are likewise correlated as a simple consequence of path tracing rules. That this model fits well, and in particular, better than a single factor model, is prima facie evidence that the relationships among the two clusters of variables — that is, $\mathrm{X}_{1}$ through $X_{3}$ and $X_{4}$ through $X_{6}-$ can be thought to be mediated by their factors. If this model is found to fit well on one group, we may be interested in the question as to whether this model fits just as well for a second group. For that to be the case, we would require, as stated earlier, tests of MI at each of the stages described previously. However, consider the below model of the true structure for the second group. 


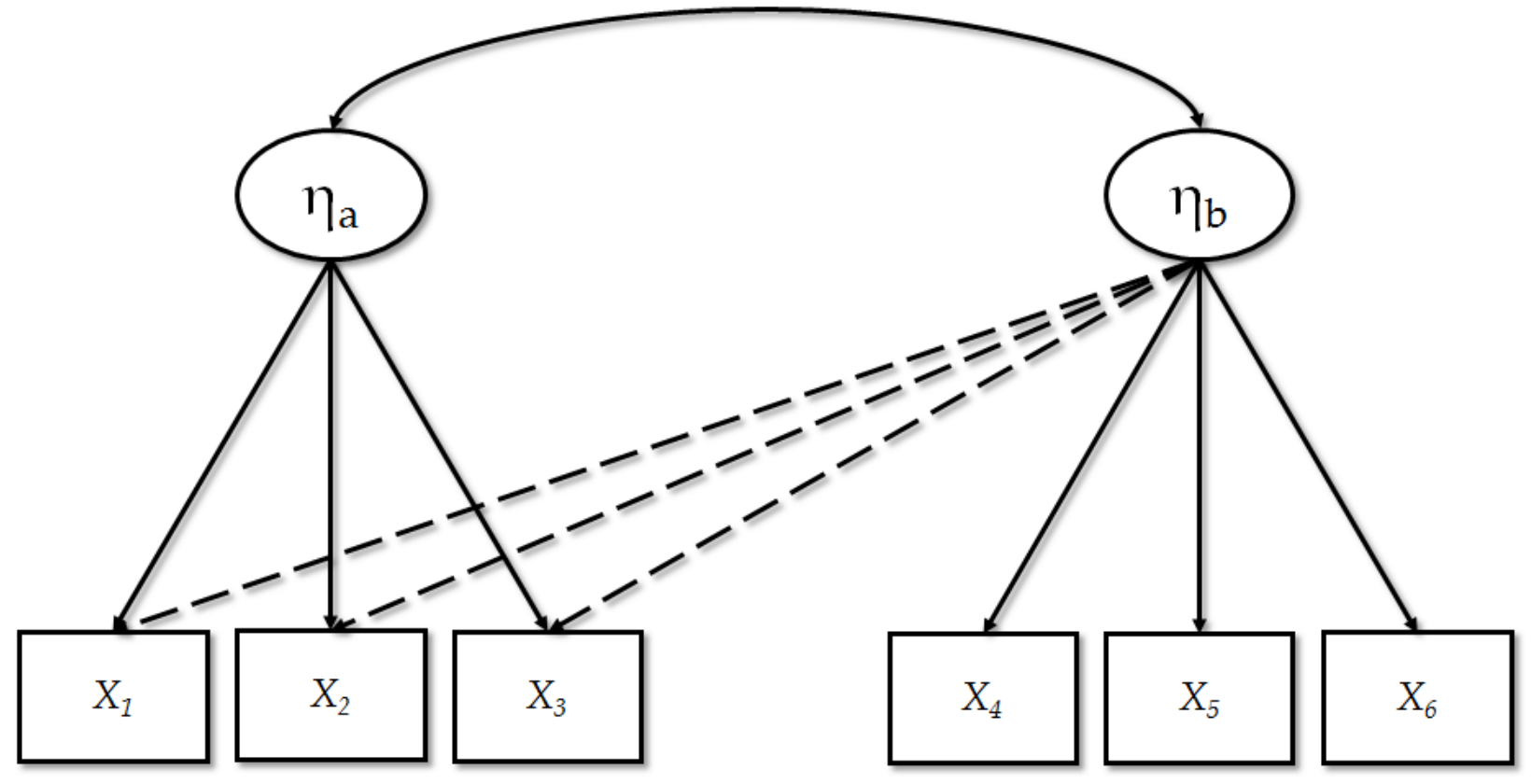

Figure II

The dashed lines in figure II are unmodeled cross-loadings which we will assume, for simplicity, are positive and equal. That is, in the second group, $\mathrm{X}_{1}, \mathrm{X}_{2}$, and $\mathrm{X}_{3}$ not only load on $\eta_{a}$ but also on the $\eta_{b}$. We will also assume that MI holds for this model, with the exception of these cross-loadings. The three group-specific cross-loadings may be unproblematic depending on their weights. If they are not exorbitantly high, they can be absorbed by the factor covariance and, as such, MI may be retained with the same modeled loadings for our two common factors. In this case, that implies that in order to absorb the increased correlations between the two clusters that results from the cross-loadings, the factor covariance must be multiplied by some constant greater than one since factor covariances are still allowed to differ under MI. This procedure obviously becomes problematic if the cross-loadings are too large, generating misfit and potentially forcing a change in the loadings and thus a violation of metric invariance if, say, the factor covariance is increased to its limit (identity). Figure II, can show a violation of local independence and a case where the common interpretation of the manifest variables is jeopardized. 
Approach to response bias in Lukaszewski et al. 's (2017).

With respect to specific varieties of response bias, the controls for bias used by Lukaszewski et al. (2017) were both atheoretical and formally inadequate. Firstly, each of their controls required an invariant effect of that variable or the form of bias it was intended to represent. For an observed variable like a score ranking items by their risk for acquiescent bias to work as a background variable capable of assuaging MI issues requires that its measurement properties are shared between groups, but, like MI more generally, this is by no means guaranteed. It is more proper to model biasing method factors as just that: factors (e.g., see Ober et al., Cheng, Jacobucci, \& Whitney, 2021). This would allow, at the very least, holding their effects constant, even though it might engender issues with multidimensionality. Additionally, assessing the effect of, say, literacy by controlling for a potentially different group's literacy rates and using the translation status of an item does not account for literacy as an explanation for differences in test functioning. The first complaint should be obvious, and especially so for the sorts of convenience samples that constitute most of the representation from poorer countries, but the second requires more explanation. Briefly, translating an item does not necessarily preserve its function among speakers of different languages; translated items can be biased for a variety of reasons. If MI is achieved, these controls are superfluous (Lubke et al., 2003); if it is not achieved and they cannot help it be achieved, they are likely uninformative.

\section{Is Cross-cultural MI Attainable for Big Five Traits?}

In addition to work indicating that the factorial structure of the Big Five is unlikely congeneric (i.e., each observed personality item loading only on its conceptually-intended factor; see Marsh et al., 2010; for cross-cultural work, see Laajaj et al., 2019), the MI of Big Five personality traits across cultures, especially those in non-western, educated, industrialized, rich, and democratic (WEIRD) populations, is far from a certainty. For example, studies in social- 
personality research directly investigating cross-cultural MI of Big Five latent models derived from well-known personality inventories (e.g., NEO-PI-R; Costa \& McCrae, 1992) have demonstrated that it often does not hold (e.g., Church et al., 2011; Gurven et al., 2013; see also Poortinga, Van De Vijver, \& Van Hemert, 2002). This is not at all surprising given that tests of MI are most frequently interrogated on a presumed congeneric measurement model, which as noted above is unlikely to be the case for Big Five personality traits (Gurven et al., 2013; Laajaj et al., 2019; Marsh et al., 2010; see also Marsh et al., 2018; Mezquita et al., 2019; Vassend \& Skrondal, 1997).

Additional evidence suggesting cross-cultural MI is unlikely to hold for big five personality traits comes from behavior genetic studies suggesting personality factors may not "exist" in the realist sense (i.e., in the sense that a common factor or 'common pathway' is the underlying cause of responses; Borsboom et al., 2003, Franić et al., 2013). While researchers employing latent variable models certainly do not have to adopt a realist stance, and can, for instance, adopt descriptivism (Jonas \& Markon, 2016; Van Bork, Wijsen, \& Rhemtulla, 2017); there is little reason to expect that relationships among latent variables and items should be conserved across cultures unless the former are causes of responses (i.e., manifest items that index the personality trait), and hence variation among them. Below we discuss personality trait research that has sought to evaluate the validity of the proportionality of influences on manifest covariances with the constraints of common factors via comparisons of common and independent pathway behavior genetic models (Franić et al., 2013; see figures III and IV below). 


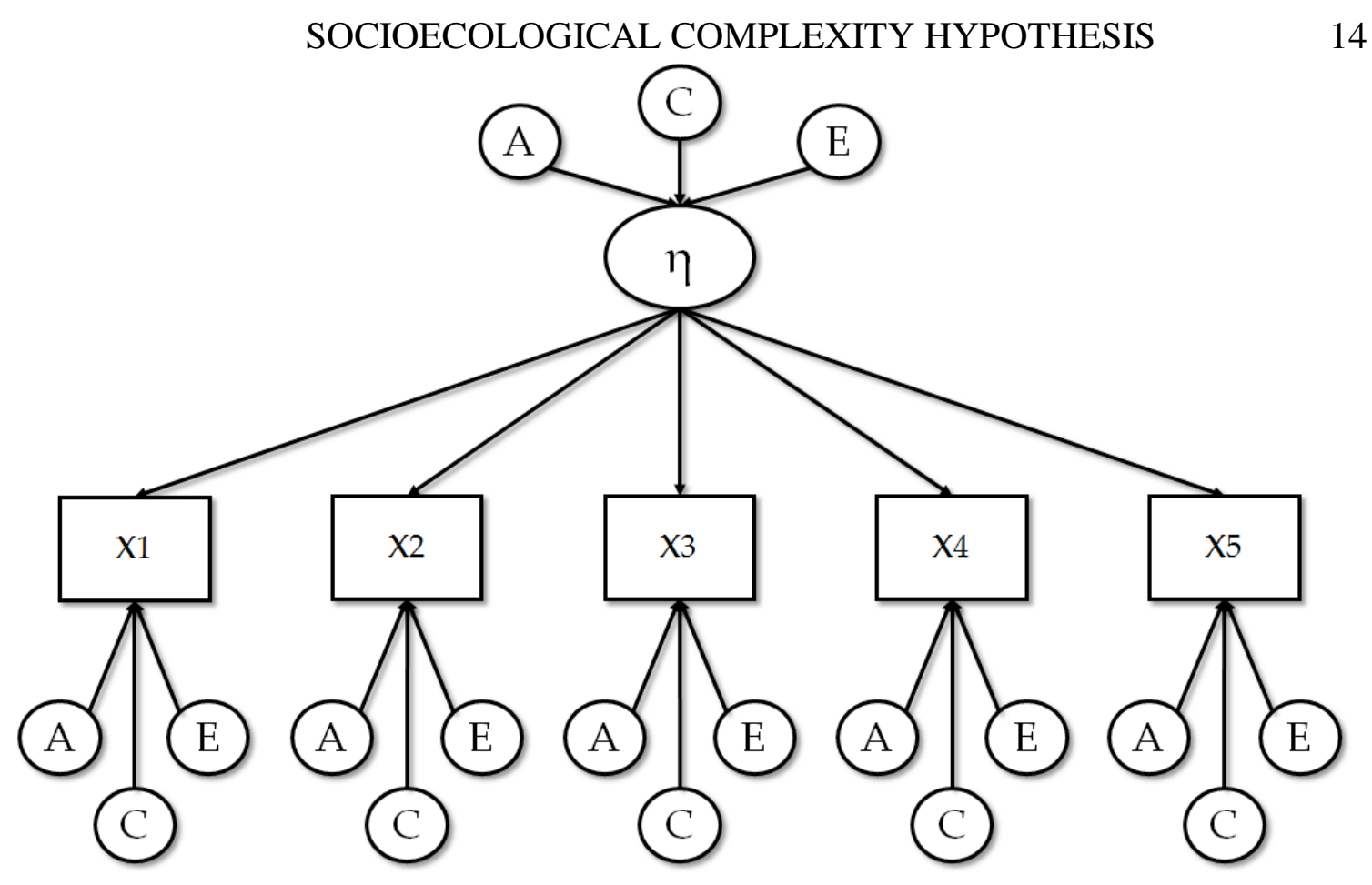

Figure III: A Common Pathway Model

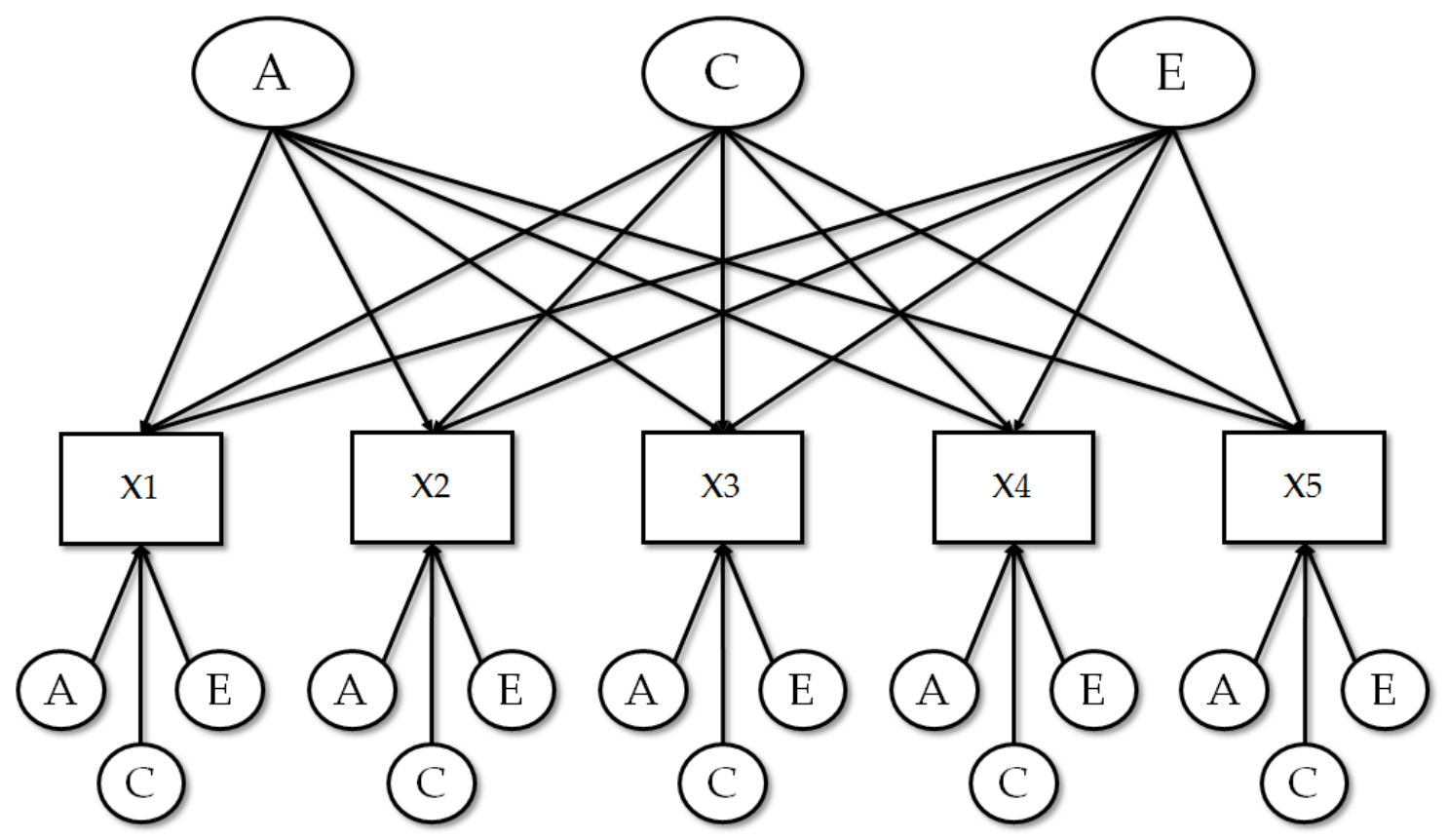

Figure IV: An Independent Pathway Model

Figures III and IV use the typical behavior genetic meanings for the A, C, and E factors. For clarity, these multigroup SEMs exploit the differences in genetic resemblance between, 
typically, monozygotic (identical) and dizygotic (fraternal) twins to identify the variance in some outcome which can be attributed to additive genetic effects, A, shared environmental effects that make siblings more similar, $\mathrm{C}$, and unique environmental effects that make siblings less similar, E. The common pathway model denotes the case where the common influences on a factor's manifest variables are consistent with the factor's proportionality constraints, suggesting it exists in the realist sense.

In contrast to the common pathway model, the independent pathway model represents the case where genetic and environmental influences on manifest response variation cannot be said to be mediated by a common latent factor. Whereas MI requires mediation of group differences by latent variables, in this case there is no latent variable. For MI to fit without a latent variable to speak of in some domain requires the effect of group membership to incidentally mirror the phenotypic factor model through the independent $\mathrm{A}, \mathrm{C}$, and $\mathrm{E}$ factors, whose residuals, but not necessarily their factor loadings (if we grant that "A" effects can mirror "C" effects, and so on), must also yield MI. This is extremely difficult to believe for the obvious reason that it implies a tremendous set of coincidences to occur. Figure V illustrates these possibilities for a multiple indicator multiple cause (MIMIC) model in which a latent variable, $\eta$, is explained by three manifest variables, socioeconomic status (SES), health and culture. Common pathway models can be thought of as a special case of the MIMIC model. 

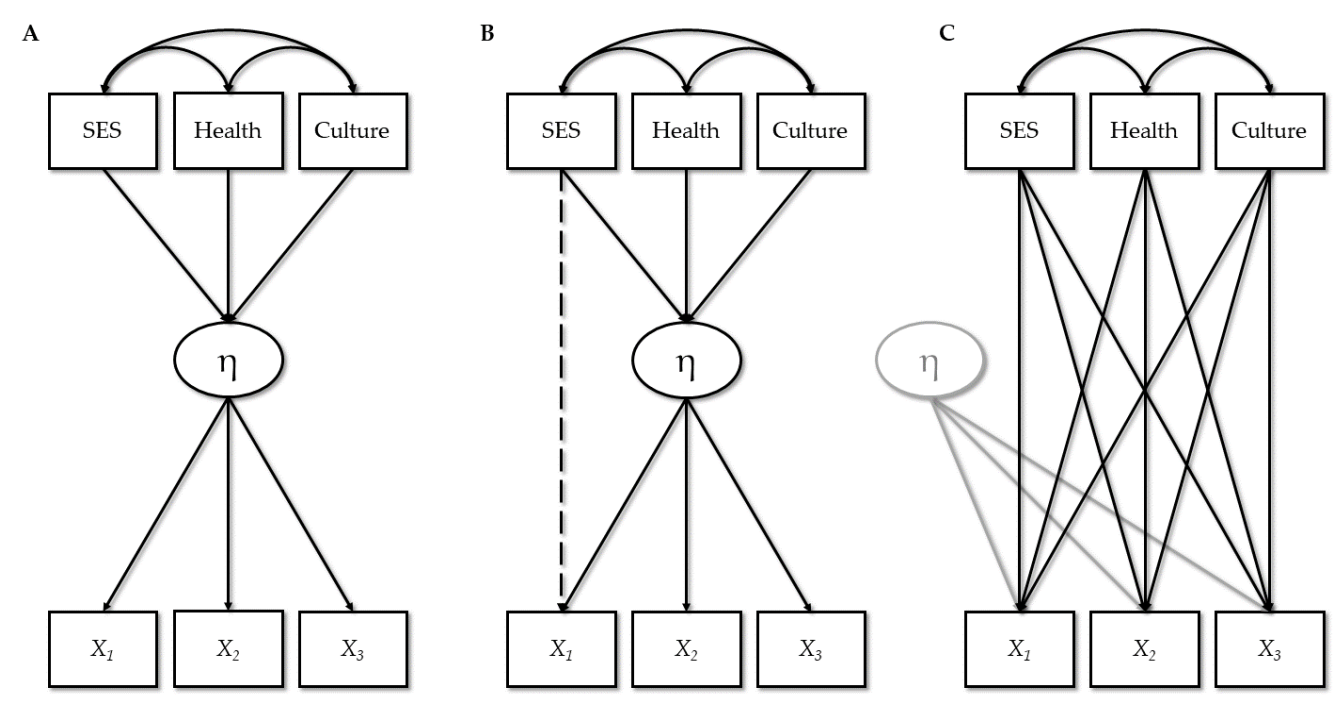

Figure V: MIMIC Models to be Considered

Model A illustrates a case in which $\eta$ fully mediates the influences of the causal variables SES, health and culture on variables $\mathrm{X}_{1}$ through $\mathrm{X}_{3}$; in this situation, it is legitimate to represent the relationships among $\mathrm{X}_{1}$ through $\mathrm{X}_{3}$ with a factor model. Model $\mathrm{B}$ is the same model for a second group. In it, an influence has an additional, unmediated effect on one of the manifest variables; in this case, MI would be untenable for the MIMIC model and the intercept would be raised in the phenotypic factor model sans causes. If the causes had multiple influences on specific manifest variables net of $\eta$, local independence would be violated and invariance of the phenotypic factor model would not hold. Model C may be a more realistic reimagining of what a given latent variable model actually represents: for some population, a latent variable recapitulates a consistent pattern of effects of different causes, providing statistical, if not substantive, evidence for $\eta$. If this structure of effects fails to replicate in a different group, MI will likely not appear. Thus, with qualification, a failure of MI might signal that a construct is improperly formulated or altogether lacking in one or multiple populations.

In the case of personality traits, common pathway models frequently fail to fit the data as well as independent pathways models (e.g., Briley \& Tucker-Drob, 2012; Franić et al., 2014; 
Lewis \& Bates, 2014). Overall, behavioral genetic evidence raises questions about the ontological status of the latent personality constructs comprising the Big Five because it suggests that rather than the personality traits reflecting valid latent constructs in the realist sense, they might be psychometric phenomena supported only by modeling the phenotypic correlations among personality trait indicators (see also Jang et al., 2002; Panizzon et al., 2014).

\section{A New Test of the Socioecological Complexity Hypothesis: Similar Concerns}

Our above concerns with Lukaszewski et al. (2017) are also relevant to a recent followup investigation of the socioecological complexity hypothesis (Durkee et al., 2020, preprint v1, 7/24/2020) in which the investigators utilized a contemporary approach to measurement invariance known as the alignment method (Asparouhov \& Muthén, 2009). With the alignment approach, measurement invariance is approximated (yielding approximate measurement invariance; see Asparouhov \& Muthén, 2009, for details). Durkee et al. (2020) drew on personality data from 115 nations gathered for the Open-Source Psychometrics Project.

A reading of the original preprint $(\mathrm{v} 1,7 / 24 / 2020)^{3}$ indicated the investigators fit a configural model to a five correlated factor representation of the IPIP based on the two-step approach to the alignment method. We attempted to fit and establish an acceptable configurally invariant model using the publicly available IPIP data provided by the authors in the OSF repository using exploratory structural equation modeling (ESEM; Asparouhov \& Muthén, 2009). However, model convergence was not attainable with a freely estimated model when all 115 nations were considered simultaneously and non-target loadings (i.e., cross-loadings) of

\footnotetext{
${ }^{3}$ In v2 of this preprint, dated 3/25/2021 per the OSF PsyArXiv preprints repository (https://psyarxiv.com/93qmp/), we found that the authors added three sentences noting that alignment does not allow cross-loadings and, related, ESEM and alignment have not been integrated; therefore, they needed to assume a simple structure (i.e., no crossloadings). This seems to miss the point that a well-fitting configural model is prerequisite to alignment, whatever the current state of things in terms of software capabilities. As we discuss later, however, the authors are incorrect and Marsh et al. (2018) did indeed integrate the capabilities of ESEM into their alignment-within-CFA approach, which allows cross-loadings.
} 
personality items on Big Five factors were allowed; the same was true for a CFA without crossloadings. This strongly suggests (at minimum) that a congeneric five correlated factor model representation of IPIP personality would not yield configural invariance across nations (e.g., Marsh et al., 2018), just as single-factor models in Lukaszewski et al. (2017) were not viable. Dr. B. Muthén remarked that the configural model must fit for investigation of approximate invariance to continue and if it does not, it does not make sense to proceed (personal correspondence, July 29, 2020).

While appearing to have recognized the conceptual logic and internal consistency of testing the socioecological complexity hypothesis using a configural MI model reflecting a correlated factors model of personality data, there remain several additional problems with using the alignment method to derive parameter estimates of personality data across nations. First, approximate invariance is not appropriate when presumed empirical support for the configural model is absent (personal correspondence, Dr. Bengt Muthén, July 29, 2020) and, relatedly, it will not produce a well-fitting global model where one is absent. Secondly, approximate invariance is only applicable for addressing relatively minor or few parameter inconsistencies and is not intended to be used to estimate each unique parameter across the grouping factor (e.g., nations). As the alignment method was applied by Durkee et al. (2020), it does not allow meaningful comparisons of model-based covariances nor is the effect of differential reliability on factor covariances accounted for.

Lastly, Durkee et al. (2020) utilized several control variables in secondary analyses of their focal tests to account for potentially biasing influences on the presumed causal force accounting for observed between-nation differences in personality trait covariation. However, corrections for bias assume weakly measurement invariant relationships with biasing variables. Tests of MI on the biasing variables were not conducted in the Durkee et al. (2020) analyses, 
however, threatening the typical interpretation of background variables in the MI framework laid out by Meredith (1993).

\section{Discussion}

During the past decade, social scientists have become painfully aware of methodological problems threatening the validity of their inferences. Among these are low power, $p$-hacking, and HARKing (Kerr, 1998; Open Science Collaboration, 2015; Smaldino \& McElreath, 2016); lack of formal theorizing (Muthukrishna \& Henrich, 2019; Smaldino, 2020); and overrepresentation of samples from undergraduate and WEIRD populations (Pollet \& Saxton, 2019; see also Gurven et al., 2013; Laajaj et al., 2019). Pertinent to the current study, inadequate correspondence between theory and measurement models (McGrath, 2005) as well as lack of measurement invariance (Dong \& Dumas, 2020; Stevanovich et al., 2017; Chen, Lau, Richardson, \& Dai, 2020) were identified as pressing problems. Evolutionary social science is no outlier when it comes to problems such as these and measurement invariance testing, in particular, is relatively rare in this literature (Wang, Chen, Dai, \& Richardson, 2018). Lack of attention to the validity of measures in evolutionary social science is important not only because measurement is the foundation of science, but also because the field often raises controversy and seems to receive a proportional degree of scrutiny (e.g., see Von Hippel, Buss, \& Richardson, 2020).

Without formal demonstration of MI across cultures on the personality trait measures they draw on, Lukaszewski et al. (2017; Durkee et al., 2020) cannot properly or reliably evaluate the evidence for the socioecological complexity hypothesis. In the strictest sense, their approach is empirically untenable since the universality of the individual personality traits themselves, nor their covariation with one another, can be supported. Moreover, even if formal allowance is made for relaxing the need for strict MI as a necessary prerequisite for the universality of personality traits (e.g., partial measurement invariance) to account for maintenance of heritable 
interindividual variation in personality trait expression (perhaps suggesting there is no universal phenotypic optimum; e.g., see Nettle, 2006) ${ }^{4}$, the sociecological niche diversity hypothesis should be rigorously contrasted with other alternative explanations for personality trait covariation both within and across societies (e.g., life history theory; see Del Giudice, 2020; see also Mededović, 2020).

\section{Suggestions for Future Work}

We conclude by offering some suggestions on what we believe may be more analytically viable ways forward if future tests of the hypothesis are undertaken. First, interrogation of measurement invariance on personality measures that are used in tests of the socioecological complexity hypothesis should proceed from the start with a configural test of a postulated correlated factors model. In addition to concerns about the true factor structure of personality factor models based on the Big Five model (e.g., Marsh et al., 2010; Mezquita et al., 2019), the Big Five Inventory (Benet-Martinez \& John, 1998) is known to have a factor structure characterized by correlated trait dimensions (Benet-Martinez \& John, 1998; Borkenau \& Ostendorf, 1990); not accounting for this in tests of configural invariance prohibits the ability to adjudicate whether any differences in personality trait covariances as a function of socioecological complexity are fully mediated by trait covariances or may be confounded with lack of configural invariance in personality trait structure across nations.

\footnotetext{
${ }^{4}$ A case for partial measurement invariance as a relaxed form of MI that still supports the notion of the universality of personality traits (i.e., 'near uniformity', Tooby \& Cosmides, 1992) would need to be clearly argued on theoretical grounds. For example, one might suggest with repeated assessments of personality (cf. Borsboom et. al., 2003) that only full intraindividual structural (metric) invariance needs to hold and that interindividual variation in some subset of freely estimated intercepts of personality trait items at the intraindividual level reflect individual reaction norms (Nettle \& Penke, 2010). These intraindividual non-invariant intercepts would be reflected in intraindividual personality trait means, and could be used to argue for the plausibility that there is no universal phenotypic optimum in Big Five personality traits (cf. Nettle, 2006) between individuals and larger groupings (e.g., nations), despite a fully invariant universal structure to the traits as reflected in their intraindividual loading patterns. Nonetheless, the impact of socioecological niche on Big Five personality trait covariances might still be examined.
} 
One potential way forward in this regard may be the use of an extension of the original alignment approach (alignment-within-CFA [AwC], Marsh et al., 2018) or ESEM to establish a viable configural model of Big Five personality traits across a subset of diverse nations reflecting a range of different cultural practices. This would be a welcome first step in a larger program of empirical scholarship on the socioecological complexity hypothesis given that to date no extensive evolutionary theory exists that explains the Five Factor Model from first principles (see Gurven et al., 2013).

A second analytic possibility worth considering is the use of a multiple group, multilevel confirmatory factor analytic approach (cf. Dyer, Hanges, \& Hall, 2005; Muthen, 1991; 1994; see also de Jong \& Steenkamp, 2007). One upshot of using an approach of this kind is that it conceptually aligns with the multilevel analytic approach used by Durkee, Lukaszewski, and Buss (2020) in their national-level investigation of the psychological foundations of human status allocation. A somewhat similar approach, though not distinguishing between within and between levels of analysis, is the moderated nonlinear factor analysis approach to MI of Bauer (2017). Within this approach, having established an optimal configural model as the approach assumes group-level configural invariance of the factor structure (see Bauer 2017, for details), one could interrogate MI and, assuming it holds at the metric and scalar levels, regress factor variance-covariance parameters on the nation-level factor. If these parameter estimates significantly vary as a function of the predictive effect of the nation-level factor (i.e., are moderated by the nation factor), evidence in favor of the socioecological complexity hypothesis would be suggested.

This paper has been mainly focused on the socioecological complexity hypothesis, but much of our advice and criticism also applies to research on constructs such as pride, shame, or individualism-collectivism. The last of these constructs is particularly ripe for a reassessment in 
the cross-cultural psychology literature. For example, the interpretation of individualismcollectivism as a continuum that different populations lie on when that is not always appropriate (see Taras et al., 2013) could be avoided if it was known that the dimensionality - as could be understood by latent variable models - does not resemble a continuum from one construct to another. Additionally, what may appear to be counterintuitive findings about cooperation and individualism-collectivism (e.g., Allik \& Realo, 2004) may be found untenable due to measurements being uninterpretable in terms of the intended constructs. The differences - not only in terms of individualism-collectivism, but in many areas - may really derive from difficultto-interpret biases in the instruments used.

Although our commentary has been critical, we agree with Mededović (2020) that cultural ecological theory has the potential to enhance our understanding about the basic functional features of personality traits and how they may be shaped by local features of the cultural environment. We also agree with Lukaszewski and colleagues that the socioecological complexity might play an important role in some aspects of personality structure. Nonetheless, attention to the conceptual and methodological issues we have identified is imperative if we are to determine the sources of trait covariance and how they may vary across groups. We hope that our review of measurement issues in extant tests of the socioecological complexity hypothesis is useful as further theory development and empirical tests of the novel sociecological complexity hypothesis are considered and undertaken.

Funding: The authors received no financial support for the work reported here.

Conflicts of interest/Competing interests: The authors report no conflicts of interest. 
Availability of data and material: N/A

Code availability: N/A

Ethics approval: N/A

Consent to participate: N/A

Consent for publication: All authors have consented to publication of this manuscript. 


\section{References}

Allik, J., \& Realo, A. (2004). Individualism-Collectivism and Social Capital. Journal of CrossCultural Psychology, 35(1), 29-49. https://doi.org/10.1177/0022022103260381

Andersen, E. B. (1977). Sufficient statistics and latent trait models. Psychometrika, 42(1), 69-81. https://doi.org/10.1007/BF02293746

Asparouhov, T., \& Muthén, B. (2009). Exploratory structural equation modeling. Structural Equation Modeling: A Multidisciplinary Journal, 16(3), 397-438.

Bauer, D. J. (2017). A more general model for testing measurement invariance and differential item functioning. Psychological Methods, 22(3), 507.

Benet-Martínez, V., \& John, O. P. (1998). Los Cinco Grandes across cultures and ethnic groups: Multitrait-multimethod analyses of the Big Five in Spanish and English. Journal of Personality and Social Psychology, 75(3), 729.

Borkenau, P., \& Ostendorf, F. (1990). Comparing exploratory and confirmatory factor analysis: A study on the 5-factor model of personality. Personality and Individual Differences, $11(5), 515-524$.

Borkenau, P., \& Ostendorf, F. (1998). The Big Five as states: How useful is the five-factor model to describe intraindividual variations over time? Journal of Research in Personality, 32(2), 202-221.

Borsboom, D., Mellenbergh, G. J., \& Van Heerden, J. (2003). The theoretical status of latent variables. Psychological Review, 110(2), 203.

Briley, D. A., \& Tucker-Drob, E. M. (2012). Broad Bandwidth or High Fidelity? Evidence from the Structure of Genetic and Environmental Effects on the Facets of the Five Factor Model. Behavior Genetics, 42(5), 743-763. https://doi.org/10.1007/s10519-012-9548-8 
Brown, T. A. (2006). Confirmatory factor analysis for applied research (pp. xiii, 475). The Guilford Press.

Brown, T. A. (2015). Confirmatory factor analysis for applied research. Guilford publications.

Chen, C.-C., Lau, J. M., Richardson, G. B., \& Dai, C.-L. (2020). Measurement Invariance Testing in Counseling. Journal of Professional Counseling: Practice, Theory \& Research, 47(2), 89-104. https://doi.org/10.1080/15566382.2020.1795806

Church, A. T., Alvarez, J. M., Mai, N. T., French, B. F., Katigbak, M. S., \& Ortiz, F. A. (2011). Are cross-cultural comparisons of personality profiles meaningful? Differential item and facet functioning in the Revised NEO Personality Inventory. Journal of Personality and Social Psychology, 101(5), 1068.

Collaboration, O. S. (2015). Estimating the reproducibility of psychological science. Science, 349(6251). https://doi.org/10.1126/science.aac4716

Collins, L. M. (2006). Analysis of longitudinal data: The integration of theoretical model, temporal design, and statistical model. Annu. Rev. Psychol., 57, 505-528.

Costa, P. T., \& McCrae, R. R. (1992). Revised NEO personality inventory (NEO-PI-R) and Neo five-factor inventory (NEO-FFI). Psychological Assessment Resources.

De Jong, M. G., Steenkamp, J.-B. E. M., \& Fox, J.-P. (2007). Relaxing Measurement Invariance in Cross-National Consumer Research Using a Hierarchical IRT Model. Journal of Consumer Research, 34(2), 260-278. https://doi.org/10.1086/518532

Del Giudice, M. (2020). Rethinking the fast-slow continuum of individual differences. Evolution and Human Behavior.

DeShon, R. P. (2004). Measures are not invariant across groups without error variance homogeneity. Psychology Science, 46, 137-149. 
Dong, Y., \& Dumas, D. (2020). Are personality measures valid for different populations? A systematic review of measurement invariance across cultures, gender, and age. Personality and Individual Differences, 160, 109956. https://doi.org/10.1016/j.paid.2020.109956

Durkee, P, Lukaszewski, A., \& Buss, D. (2020). Psychological foundations of human status allocation. Proceedings of the National Academy of Sciences, 117(35), 21235-21241.

Durkee, P., Lukaszewski, A., von Rueden, C., Gurven, M., Buss, D. M., \& Tucker-Drob, E. (2020). Niche diversity predicts personality structure across 115 nations.

Dyer, N. G., Hanges, P. J., \& Hall, R. J. (2005). Applying multilevel confirmatory factor analysis techniques to the study of leadership. The Leadership Quarterly, 16(1), 149-167.

Edwards, J. R., \& Bagozzi, R. P. (2000). On the nature and direction of relationships between constructs and measures. Psychological Methods, 5(2), 155-174.

Franić, S., Borsboom, D., Dolan, C. V., \& Boomsma, D. I. (2014). The Big Five Personality Traits: Psychological Entities or Statistical Constructs? Behavior Genetics, 44(6), 591604. https://doi.org/10.1007/s10519-013-9625-7

Franić, S., Dolan, C. V., Borsboom, D., Hudziak, J. J., van Beijsterveldt, C. E., \& Boomsma, D. I. (2013). Can genetics help psychometrics? Improving dimensionality assessment through genetic factor modeling. Psychological Methods, 18(3), 406.

Gorsuch, R. L. (1988b). Exploratory factor analysis. In Handbook of multivariate experimental psychology, 2nd ed (pp. 231-258). Plenum Press. https://doi.org/10.1007/978-1-46130893-5_6

Gurven, M., Von Rueden, C., Massenkoff, M., Kaplan, H., \& Lero Vie, M. (2013). How universal is the Big Five? Testing the five-factor model of personality variation among 
forager-farmers in the Bolivian Amazon. Journal of Personality and Social Psychology, $104(2), 354$.

Hu, L., \& Bentler, P. M. (1999). Cutoff criteria for fit indexes in covariance structure analysis: Conventional criteria versus new alternatives. Structural Equation Modeling: A Multidisciplinary Journal, 6(1), 1-55.

Jang, K. L., Livesley, W. J., Angleitner, A., Riemann, R., \& Vernon, P. A. (2002). Genetic and environmental influences on the covariance of facets defining the domains of the fivefactor model of personality. Personality and Individual Differences, 33(1), 83-101.

Jonas, K. G., \& Markon, K. E. (2016). A descriptivist approach to trait conceptualization and inference. Psychological Review, 123(1), 90.

Kerr, N. L. (1998). HARKing: Hypothesizing After the Results are Known. Personality and Social Psychology Review, 2(3), 196-217. https://doi.org/10.1207/s15327957pspr0203_4

Laajaj, R., Macours, K., Hernandez, D. A. P., Arias, O., Gosling, S. D., Potter, J., Rubio-Codina, M., \& Vakis, R. (2019). Challenges to capture the big five personality traits in nonWEIRD populations. Science Advances, 5(7), eaaw5226.

Lewis, G. J., \& Bates, T. C. (2014). How genes influence personality: Evidence from multi-facet twin analyses of the HEXACO dimensions. Journal of Research in Personality, 51, 9-17. https://doi.org/10.1016/j.jrp.2014.04.004

Liang, X., \& Luo, Y. (2020). A Comprehensive Comparison of Model Selection Methods for Testing Factorial Invariance. Structural Equation Modeling: A Multidisciplinary Journal, 27(3), 380-395. https://doi.org/10.1080/10705511.2019.1649983

Lubke, G. H., Dolan, C. V., Kelderman, H., \& Mellenbergh, G. J. (2003). Weak measurement invariance with respect to unmeasured variables: An implication of strict factorial 
invariance. British Journal of Mathematical and Statistical Psychology, 56(2), 231-248. https://doi.org/10.1348/000711003770480020

Lukaszewski, A. W., Gurven, M., von Rueden, C. R., \& Schmitt, D. P. (2017). What explains personality covariation? A test of the socioecological complexity hypothesis. Social Psychological and Personality Science, 8(8), 943-952.

Marsh, H. W., Guo, J., Parker, P. D., Nagengast, B., Asparouhov, T., Muthén, B., \& Dicke, T. (2018). What to do when scalar invariance fails: The extended alignment method for multi-group factor analysis comparison of latent means across many groups. Psychological Methods, 23(3), 524.

Marsh, H. W., Lüdtke, O., Muthén, B., Asparouhov, T., Morin, A. J., Trautwein, U., \& Nagengast, B. (2010). A new look at the big five factor structure through exploratory structural equation modeling. Psychological Assessment, 22(3), 471.

McGrath, R. E. (2005). Conceptual complexity and construct validity. Journal of Personality Assessment, 85(2), 112-124. https://doi.org/10.1207/s15327752jpa8502_02

Mededović, J. (2020). An Evolutionary Ecological Framework for Understanding Human Behavioral Syndromes: Commentary on Lukaszewski et al. 2017. Social Psychological and Personality Science, 11(4), 570-573.

Mellenbergh, G. J. (1989). Item bias and item response theory. International Journal of Educational Research, 13(2), 127-143. https://doi.org/10.1016/0883-0355(89)90002-5

Meredith, W. (1993). Measurement invariance, factor analysis and factorial invariance. Psychometrika, 58(4), 525-543.

Meredith, W. (1995). Two wrongs may not make a right. Multivariate Behavioral Research, 30(1), 89-94. 
Meredith, W., \& Teresi, J. A. (2006). An essay on measurement and factorial invariance. Medical Care, S69-S77.

Mezquita, L., Bravo, A. J., Morizot, J., Pilatti, A., Pearson, M. R., Ibáñez, M. I., Ortet, G., \& Team, C.-C. A. S. (2019). Cross-cultural examination of the Big Five Personality Trait Short Questionnaire: Measurement invariance testing and associations with mental health. PloS One, 14(12), e0226223.

Muthén, B. O. (1991). Multilevel factor analysis of class and student achievement components. Journal of Educational Measurement, 28(4), 338-354.

Muthén, B. O. (1994). Multilevel covariance structure analysis. Sociological Methods \& Research, 22(3), 376-398.

Muthukrishna, M., \& Henrich, J. (2019). A problem in theory. Nature Human Behaviour, 3(3), $221-229$.

Nettle, D. (2006). The evolution of personality variation in humans and other animals. American Psychologist, 61(6), 622.

Nettle, D., \& Penke, L. (2010). Personality: Bridging the literatures from human psychology and behavioural ecology. Philosophical Transactions of the Royal Society B: Biological Sciences, 365(1560), 4043-4050.

Ober, T. M., Cheng, Y., Jacobucci, R., \& Whitney, B. M. (2020). Examining the factor structure of the Big Five Inventory-2 personality domains with an adolescent sample.

\section{Psychological Assessment.}

Panizzon, M. S., Vuoksimaa, E., Spoon, K. M., Jacobson, K. C., Lyons, M. J., Franz, C. E., Xian, H., Vasilopoulos, T., \& Kremen, W. S. (2014). Genetic and Environmental Influences of General Cognitive Ability: Is g a valid latent construct? Intelligence, 43, 
65-76. https://doi.org/10.1016/j.intell.2014.01.008

Pokropek, A., Davidov, E., \& Schmidt, P. (2019). A monte carlo simulation study to assess the appropriateness of traditional and newer approaches to test for measurement invariance. Structural Equation Modeling: A Multidisciplinary Journal, 26(5), 724-744.

Pollet, T. V., \& Saxton, T. K. (2019). How Diverse Are the Samples Used in the Journals 'Evolution \& Human Behavior' and 'Evolutionary Psychology'? Evolutionary Psychological Science, 5(3), 357-368. https://doi.org/10.1007/s40806-019-00192-2

Poortinga, Y. H., Van De Vijver, F. J., \& Van Hemert, D. A. (2002). Cross-cultural equivalence of the Big Five. In The five-factor model of personality across cultures (pp. 281-302). Springer.

Raykov, T. (2004). Behavioral scale reliability and measurement invariance evaluation using latent variable modeling. Behavior Therapy, 35(2), 299-331.

Rozin, P. (2001). Social Psychology and Science: Some Lessons From Solomon Asch. Personality and Social Psychology Review, 5(1), 2-14. https://doi.org/10.1207/S15327957PSPR0501_1

Sluis, S. van der, Verhage, M., Posthuma, D., \& Dolan, C. V. (2010). Phenotypic Complexity, Measurement Bias, and Poor Phenotypic Resolution Contribute to the Missing Heritability Problem in Genetic Association Studies. PLOS ONE, 5(11), e13929.

Smaldino, P. E. (2020). How to Build A Strong Theoretical Foundation. PsyArXiv. https://doi.org/10.31234/osf.io/khrdx

Smaldino, P. E., Lukaszewski, A., von Rueden, C., \& Gurven, M. (2019). Niche diversity can explain cross-cultural differences in personality structure. Nature Human Behaviour, 3(12), 1276-1283. 
Smaldino, P. E., \& McElreath, R. (2016). The natural selection of bad science. Royal Society Open Science, 3(9), 160384. https://doi.org/10.1098/rsos.160384

Stevanovic, D., Jafari, P., Knez, R., Franic, T., Atilola, O., Davidovic, N., Bagheri, Z., \& Lakic, A. (2017). Can we really use available scales for child and adolescent psychopathology across cultures? A systematic review of cross-cultural measurement invariance data. Transcultural Psychiatry, 54(1), 125-152. https://doi.org/10.1177/1363461516689215

Taras, V., Sarala, R., Muchinsky, P., Kemmelmeier, M., Singelis, T. M., Avsec, A., Coon, H. M., Dinnel, D. L., Gardner, W., Grace, S., Hardin, E. E., Hsu, S., Johnson, J., Karakitapoğlu Aygün, Z., Kashima, E. S., Kolstad, A., Milfont, T. L., Oetzel, J., Okazaki, S., ... Sinclair, H. C. (2014). Opposite Ends of the Same Stick? Multi-Method Test of the Dimensionality of Individualism and Collectivism. Journal of Cross-Cultural Psychology, 45(2), 213-245. https://doi.org/10.1177/0022022113509132

Thurstone, L. L. (1931). Multiple factor analysis. Psychological Review, 38(5), 406.

Thurstone, L. L. (1947). Multiple-factor analysis; a development and expansion of The Vectors of Mind. Tooby, J., \& Cosmides, L. (1992). The psychological foundations of culture. In J. Barkow, L. Cosmides, \& J. Tooby (Eds.), The adapted mind: Evolutionary psychology and the generation of culture (pp. 19-136). Oxford University Press.

Tóth-Király, I., Bõthe, B., Rigó, A., \& Orosz, G. (2017). An illustration of the exploratory structural equation modeling (ESEM) framework on the passion scale. Frontiers in Psychology, 8, 1968.

Van Bork, R., Wijsen, L. D., \& Rhemtulla, M. (2017). Toward a causal interpretation of the common factor model. Disputatio, 9(47), 581-601. 
Van de Schoot, R., Lugtig, P., \& Hox, J. (2012). A checklist for testing measurement invariance. European Journal of Developmental Psychology, 9(4), 486-492.

Vassend, O., \& Skrondal, A. (1997). Validation of the NEO Personality Inventory and the fivefactor model. Can findings from exploratory and confirmatory factor analysis be reconciled? European Journal of Personality, 11(2), 147-166.

von Hippel, W., Buss, D. M., \& Richardson, G. B. (2020). Science progresses through open disagreement: Rejoinder to Fine (2020). Archives of Scientific Psychology, 8(1), 11. https://doi.org/10.1037/arc0000073

Wang, S., Chen, C.-C., Dai, C.-L., \& Richardson, G. B. (2018). A Call for, and Beginner's Guide to, Measurement Invariance Testing in Evolutionary Psychology. Evolutionary Psychological Science, 4(2), 166-178. https://doi.org/10.1007/s40806-017-0125-5 